\title{
Special feature: the global energy transformation and human survivability
}

\author{
Yuichi Ikeda ${ }^{1} \cdot$ Dimiter Ialnazov ${ }^{1}$
}

Published online: 26 April 2017

(C) Japan Association for Evolutionary Economics 2017

The Graduate School of Advanced Integrated Studies in Human Survivability (GSAIS), Kyoto University, has been organizing international symposia every year since its inception in April 2013. The international symposia are held in line with the GSAIS' mission to develop young future leaders who can deal with the complex global challenges facing humanity in areas such as energy, water, food, population, diseases, etc.

The Fourth International Symposium "The Global Energy Transformation: a Quest for Solutions from the Perspective of Human Survivability" was held on 19-20 November 2015. The symposium was held at an important juncture of world history: between the adoption of the Sustainable Development Goals (SDGs) in New York in September and the UN's Climate Change Conference (COP 21) in Paris in December 2015. Both events could be seen as attempts to take action in response to the above-mentioned global challenges. For instance, to limit the increase of global temperatures to less than two degrees Celsius compared to preindustrial levels, the participants in the COP 21 in Paris agreed to reduce greenhouse gas (GHG) emissions until 2030. There is also a wide consensus that to reduce GHG emissions, the world needs to shift to cleaner energy sources.

The symposium was supported by the program for leading graduate schools (allround type) project by the Ministry of Education, Culture, Sports, Science and Technology-Japan (MEXT). The associate editor of Evolutionary and Institutional Economics Review (YI) and the chief organizer of the symposium (DI), are grateful to Professor Masao Kitano, Executive Vice-President for Education, Information Infrastructure, and Evaluation, Kyoto University, and to Professor Shuichi Kawai, Dean of GSAIS, Kyoto University. We wish to express our thanks to the other members of the organizing committee: Professor Eriko Ishida Kawai (GSAIS,

Yuichi Ikeda

ikeda.yuichi.2w@kyoto-u.ac.jp

1 GSAIS, Kyoto University, Sakyo-ku, Kyoto 606-8306, Japan 
Kyoto University), Professor Yosuke Yamashiki (GSAIS, Kyoto University), Professor Eiichi Yamaguchi (GSAIS, Kyoto University), and Associate Professor Marc-Henri Deroche (GSAIS, Kyoto University). We also express our thanks to Professor Yuji Aruka (Chuo University), the staff members of Springer Japan, and the members of the conference secretariat in GSAIS, Kyoto University. Finally, we would like to thank all authors for their contributions to this special feature of Evolutionary and Institutional Economics Review. 